\title{
Mixed convection in non-Newtonian fluids along nonisothermal horizontal surfaces in porous media
}

\author{
Rama Subba Reddy Gorla, K. Shanmugam, M. Kumari
}

\begin{abstract}
A nonsimilar boundary layer analysis is presented for the problem of mixed convection in power-law type non-Newtonian fluids along horizontal surfaces with variable wall temperature distribution. The mixed convection regime is divided into two regions, namely, the forced convection dominated regime and the free convection dominated regime. The two solutions are matched. Numerical results are presented for the details of the velocity and temperature fields. A discussion is provided for the effect of viscosity index on the surface heat transfer rate.

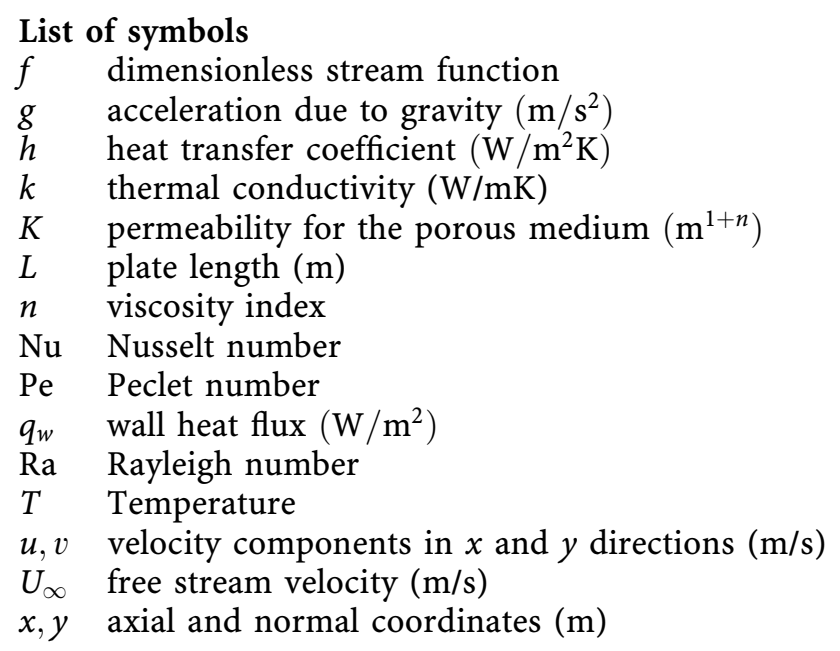

\section{Greek symbols}

effective thermal diffusivity of porous medium $\left(\mathrm{m}^{2} / \mathrm{s}\right)$ volumetric coefficient of thermal expansion $(1 / \mathrm{K})$ similarity variable dimensionless temperature
\end{abstract}

Rama Subba Reddy Gorla

K. Shanmugam

Department of Mechanical Engineering

Cleveland State University

Cleveland, Ohio 44115, USA

M. Kumari

Department of Mathematics

Indian Institute of Science

Bangalore 560 012, India

Correspondence to: R. S. R. Gorla kinematic viscosity $\left(\mathrm{m}^{2} / \mathrm{s}\right)$

nonsimilar parameter

density of fluid $\left(\mathrm{kg} / \mathrm{m}^{3}\right)$

consistency index for viscosity $\left(\mathrm{Ns} / \mathrm{m}^{2}\right)$

wall shear stress $(\mathrm{Pa})$

stream function

\author{
Subscripts \\ $w \quad$ wall conditions \\ $\infty \quad$ free stream conditions
}

1

\section{Introduction}

Convective heat transfer from impermeable surfaces embedded in porous media has numerous thermal engineering applications such as geothermal systems, crude oil extraction, thermal insulation and ground water pollution. Cheng $[1,2]$ presented similarity solutions for mixed convection from horizontal plates and cylinders in a fluidsaturated porous medium. Nakayama and Koyama [3] considered similarity solutions for two-dimensional and axisymmetric bodies in a porous medium. Cheng and Minkowycz [4] presented similarity solutions for free convective heat transfer from a vertical plate in a fluidsaturated porous medium. Gorla and co-workers [5-7] solved the nonsimilar problem of free convective heat transfer from a vertical plate embedded in a saturated porous medium with an arbitrarily varying surface temperature or heat flux. Aldoss et al. [8] presented solutions for mixed convection in porous media. All these studies were concerned with Newtonian fluid flows. A number of industrially important fluids including fossil fuels which may saturate underground beds display non-Newtonian behaviour. Non-Newtonian fluids exhibit a nonlinear relationship between shear stress and shear rate.

Chen and Chen [9] presented similarity solutions for free convection of non-Newtonian fluids over vertical surfaces in porous media. Nakayama and Koyama [10] studied the natural convection over a non-isothermal body of arbitrary shape embedded in a porous medium.

The present work has been undertaken in order to analyze the mixed convection from a horizontal plate in nonNewtonian fluid saturated porous media. This work finds application in many areas of engineering such as in petroleum drilling, for the effect of mixed convection of nonNewtonian fluids in a porous medium adjacent to the heated surface. The governing equations are first transformed into a dimensionless form and the resulting nonsimilar set of equations is solved by a finite difference 
method. Numerical results are presented for some representative values of the viscosity index.

\section{2}

\section{Analysis}

Let us consider the mixed convention in a porous medium from an impermeable horizontal plate at the bottom, which is heated and has a variable wall temperature. The properties of the fluid and the porous medium are assumed to be constant and isotropic. The Darcy model is considered which is valid under conditions of small pores of porous medium and flow velocity. Also, the slip velocity at the wall is imposed, which has a smaller effect on the heat transfer results as the distance from the leading edge increases. The axial and normal coordinates are $x$ and $y$, and the corresponding flow velocities are $u$ and $v$ respectively. Figure 1 shows the coordinate system and model of the flow. The gravitational acceleration $g$ is acting downwards opposite to the normal coordinate $y$. The governing equations under the Boussinesq and boundary layer approximations are given by,

$\frac{\partial u}{\partial x}+\frac{\partial v}{\partial y}=0$

$\frac{\partial u^{n}}{\partial y}=-\frac{\rho K g \beta}{\mu} \frac{\partial T}{\partial x}$

$u \frac{\partial T}{\partial x}+v \frac{\partial T}{\partial y}=\alpha \frac{\partial^{2} T}{\partial y^{2}}$

In the above equations, $T$ is the temperature of the wall; $n$ is the viscosity index; $\rho$ is the density; $K$ is the permeability of porous medium; $\beta$ is the volumetric coefficient of thermal expansion; $\mu$ is the viscosity; $\alpha$ is the equivalent thermal diffusivity of the porous medium. With power law variation in wall temperature, the boundary conditions can be written as

$y=0: v=0,\left(T-T_{\infty}\right)=A x^{\lambda}$

$y=\infty: u=U_{\infty}, T=T_{\infty}$

where $A$ and $\lambda$ are prescribed constants. Note that $\lambda=0$ corresponds to the case of uniform wall temperature.

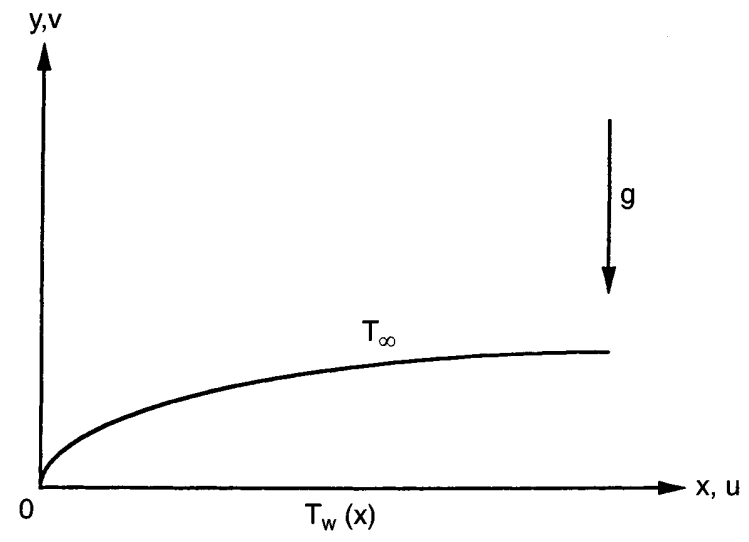

Fig. 1. Coordinate system and flow model

\section{1}

\section{Forced convection dominated regime}

The continuity equation is automatically satisfied by defining a stream function $\psi(x, y)$ such that

$u=\frac{\partial \psi}{\partial y}$ and $v=-\frac{\partial \psi}{\partial x}$

Proceeding with the analysis, we define the following transformations:

$\eta=\frac{y}{x} \mathrm{Pe}_{x}^{1 / 2}$

$\psi=\alpha \mathrm{Pe}_{x}^{1 / 2} f\left(\xi_{f}, \eta\right)$

$\xi_{f}=\frac{\operatorname{Ra}_{x}^{n}}{\operatorname{Pe}_{x}^{\frac{2 n+1}{2}}}$

$\theta=\frac{T-T_{\infty}}{T_{w}-T_{\infty}}$

$\mathrm{Pe}_{x}=\frac{U_{\infty} x}{\alpha}$

$\mathrm{Ra}_{x}=\frac{x}{\alpha}\left(\frac{\rho K g \beta \Delta T_{w}}{\mu}\right)^{1 / n}$

The governing equations and boundary conditions, Eqs. (1)-(4), can then be transformed into

$$
\begin{aligned}
& n\left(f^{\prime}\right)^{n-1} f^{\prime \prime}=-\xi_{f}\left[\lambda \theta+\left(\frac{2 \lambda-1}{2}\right) \xi_{f} \frac{\partial \theta}{\partial \xi_{f}}-\frac{\eta}{2} \theta^{\prime}\right] \\
& \theta^{\prime \prime}-\lambda f^{\prime} \theta+\frac{f \theta^{\prime}}{2}=\left(\frac{2 \lambda-1}{2}\right) \xi_{f}\left[f^{\prime} \frac{\partial \theta}{\partial \xi_{f}}-\theta^{\prime} \frac{\partial f}{\partial \xi_{f}}\right] \\
& f\left(\xi_{f}, 0\right)+(2 \lambda-1) \xi_{f} \frac{\partial f}{\partial \xi_{f}}\left(\xi_{f}, 0\right)=0 \text { or } f\left(\xi_{f}, 0\right)=0 \\
& \theta\left(\xi_{f}, 0\right)=1, \quad f^{\prime}\left(\xi_{f}, \infty\right)=1, \quad \theta\left(\xi_{f}, \infty\right)=0
\end{aligned}
$$

The primes in the above equations denote partial differentiations with respect to $\eta$. The presence of $\frac{\partial}{\partial \xi_{f}}$ in these equations makes them nonsimilar.

In the above system of equations, the dimensionless parameter $\xi_{f}$ is a measure of the buoyancy effect on forced convection. The case of $\xi_{f}=0$ corresponds to pure forced convection. The limiting case of $\xi_{f}=\infty$ corresponds to pure free convection region. The Eqs. (6)-(8) cannot be solved for the entire regime of mixed convection because of singularity at $\xi_{f}=\infty$. The above system of equations is used to solve the region covered by $\xi_{f}=0-1$ to provide the first half of the total solution of the mixed convection regime.

Some of the physical quantities of interest include the velocity components $u$ and $v$ in the $x$ and $y$ directions, the local friction factor $C_{f x}$ (defined as $\tau_{w} /\left[\left(\rho U_{\infty}^{2}\right) / 2\right]$ where $\tau_{w}=\mu(\partial u / \partial y)_{y=0}^{n}$ and the local Nusselt number $\mathrm{Nu}_{x}=h x / k$, where $h=q_{w} /\left[T_{w}(x)-T_{\infty}\right]$. They are given by

$u=u_{\infty} f^{\prime}\left(\xi_{f}, \eta\right)$ 


$$
\begin{gathered}
v=-\left(\frac{\alpha}{x}\right) \mathrm{Pe}_{x}^{1 / 2}\left(\frac{1}{2} f\left(\xi_{f}, \eta\right)-\frac{1}{2} \eta f^{\prime}\left(\xi_{f}, \eta\right)\right. \\
\left.+\left(\frac{2 \lambda-1}{2}\right) \xi \frac{\partial f}{\partial \xi}\right) \\
C_{f x}=\frac{2 \mathrm{Pe}_{x}^{n / 2}}{\operatorname{Re}_{x}}\left[f^{\prime \prime}\left(\xi_{f}, 0\right)\right]^{n}
\end{gathered}
$$

where

$\operatorname{Re}_{x}=\left(\frac{U_{\infty}^{2-n} x^{n}}{v}\right)$

$\mathrm{Nu}_{x}=-\mathrm{Pe}_{x}^{1 / 2} \theta^{\prime}\left(\xi_{f}, 0\right)$

\section{2}

\section{Free convection dominated regime}

For buoyancy dominated regime the following dimensionless variables are introduced in the transformation

$\eta=\frac{y}{x}\left(\mathrm{Ra}_{x}\right)^{\frac{n}{2 n+1}}$

$\xi_{n}=\frac{\mathrm{Pe}_{x}}{\mathrm{Ra}_{x}^{2 / 3}}$

$\psi=\alpha\left(\operatorname{Ra}_{x}\right)^{\frac{n}{2 n+1}} f\left(\xi_{n}, \eta\right)$

$\theta\left(\xi_{n}, \eta\right)=\left(\frac{T-T_{\infty}}{T_{w}(x)-T_{\infty}}\right)$

Substituting Eqs. (14) and (15) into the governing Eqs. (1)-(4) leads to

$$
\begin{aligned}
& n\left(f^{\prime}\right)^{n-1} f^{\prime \prime}+\lambda \theta+\frac{\lambda-n-1}{(2 n+1)} \eta \theta^{\prime} \\
& \quad+\frac{1-2 \lambda}{(2 n+1)} \xi_{n} \frac{\partial \theta}{\partial \xi}=0 \\
& \theta^{\prime \prime}+\frac{\lambda+n}{(2 n+1)} f \theta^{\prime}-\lambda f^{\prime} \theta \\
& \quad=\left(\frac{1-2 \lambda}{2 n+1}\right) \xi_{n}\left[f^{\prime} \frac{\partial \theta}{\partial \xi_{n}}-\theta^{\prime} \frac{\partial f}{\partial \xi_{n}}\right] \\
& (\lambda+n) f\left(\xi_{n}, 0\right)+(1-2 \lambda) \xi_{n} \frac{\partial f}{\partial \xi_{n}}\left(\xi_{n}, 0\right)=0 \text { or } \\
& f\left(\xi_{n}, 0\right)=0, \quad \theta\left(\xi_{n}, 0\right)=1, \\
& f^{\prime}\left(\xi_{n}, \infty\right)=\xi_{n}, \quad \theta\left(\xi_{n}, \infty\right)=0
\end{aligned}
$$

and the primes in Eqs. (16)-(18) denote partial differentiations with respect to $\eta$.

Note that the $\xi_{n}$ parameter here represents the forced flow effect on free convection. The case of $\xi_{n}=0$ corresponds to pure free convection and the limiting case of $\xi_{n}=\infty$ corresponds to pure forced convection. The above system of Eqs. (16)-(18) is solved over the region covered by $\xi_{n}=0-1$ to provide the other half of the solution for the entire mixed convection regime.

The velocity components $u$ and $v$, the local friction factor and the local Nusselt number for this case have the following expressions

$$
\begin{aligned}
& u=C D x^{\frac{2 \lambda-1}{2 n+1}} f^{\prime} \\
& v=-D x^{\frac{\lambda-n-1}{(2 n+1)}}\left(\frac{\lambda+n}{(2 n+1)} f\left(\xi_{n}, \eta\right)+\frac{\lambda-n-1}{(2 n+1)} \eta f^{\prime}\left(\xi_{n}, \eta\right)\right. \\
& \left.+\frac{1-2 \lambda}{(2 n+1)} \xi_{n} \frac{\partial f}{\partial \xi_{n}}\right) \\
& C_{f x}=\frac{2 \mu}{\rho U_{\infty}^{2}}\left(C^{2} D\right)^{n} x^{\frac{3 \lambda-n-2}{(2 n+1)}}\left[f^{\prime \prime}\left(\xi_{n}, 0\right)\right]^{n} \\
& \mathrm{Nu}_{x}=-\mathrm{Ra} \stackrel{(2 n+1)}{n}^{\prime}\left(\xi_{n}, 0\right)
\end{aligned}
$$

where

$$
C=\left(\frac{\rho K g \beta A}{\mu^{*} \alpha^{n}}\right)^{\frac{1}{(2 n+1)}}
$$

and

$$
D=\alpha^{\frac{n+1}{(2 n+1)}}\left(\frac{\rho K g \beta A}{\mu^{*}}\right)^{\frac{1}{(2 n+1)}}
$$

\section{3}

\section{Numerical scheme}

The numerical scheme to solve Eqs. (6) and (7) adopted here is based on a combination of the following concepts:

(a) The boundary conditions for $\eta=\infty$ are replaced by

$f^{\prime}\left(\xi, \eta_{\max }\right)=1, \quad \theta\left(\xi, \eta_{\max }\right)=0$

where $\eta_{\max }$ is a sufficiently large value of $\eta$ at which the boundary conditions (8) are satisfied. $\eta_{\max }$ varies with the value of $n$. In the present work, a value of $\eta_{\max }=25$ was checked to be sufficient for free stream behaviour.

(b) The two-dimensional domain of interest $(\xi, \eta)$ is discretized with an equispaced mesh in the $\xi$-direction and another equispaced mesh in the $\eta$-direction.

(c) The partial derivatives with respect to $\eta$ are evaluated by the second order difference approximation.

(d) Two iteration loops based on the successive substitution are used because of the nonlinearity of the equations.

(e) In each inner iteration loop, the value of $\xi$ is fixed while each of the Eqs. (6) and (7) is solved as a linear second order boundary value problem of ODE on the $\eta$ domain. The inner iteration is continued until the nonlinear solution converges with a convergence criterion of $10^{-6}$ in all cases for the fixed value of $\xi$.

(f) In the outer iteration loop, the value of $\xi$ is advanced. The derivatives with respect to $\xi$ are updated after every outer iteration step.

In the inner iteration step, the finite difference approximation for Eqs. (6) and (7) is solved as a boundary value problem. We consider Eq. (6) first. By defining $f=\phi$, Eq. (6) may be written in the form

$a_{1} \phi^{\prime \prime}+b_{1} \phi^{\prime}+c_{1} \phi=S_{1}$

where

$$
\begin{aligned}
& a_{1}=n\left|\phi^{\prime}\right|^{n-1} \\
& b_{1}=c_{1}=0
\end{aligned}
$$


$S_{1}=-\xi\left[\lambda \theta+\left(\frac{2 \lambda-1}{2}\right) \xi \frac{\partial \theta}{\partial \xi}-\frac{\eta}{2} \theta^{\prime}\right]$

The coefficients $a_{1}, b_{1}, c_{1}$ and the source term in Eq. (26) in the inner iteration step are evaluated by using the solution from the previous iteration step. Equation (26) is then transformed to a finite difference equation by applying the central difference approximations to the first and second derivatives. The finite difference equations form a tridiagonal system and can be solved by the tridiagonal solution scheme.

Equation (7) is also written as a second-order boundary value problem similar to Eq. (27), namely

$a_{2} \theta^{\prime \prime}+b_{2} \theta^{\prime}+c_{2} \theta=S_{2}$

where

$$
\begin{aligned}
& a_{2}=1 \\
& b_{2}=\frac{\phi}{2} \\
& c_{2}=-\lambda \phi^{\prime} \\
& S_{2}=\left(\frac{2 \lambda-1}{2}\right) \xi\left[\phi^{\prime} \frac{\partial \theta}{\partial \xi}-\theta^{\prime} \frac{\partial \phi}{\partial \xi}\right]
\end{aligned}
$$

The gradients

$\frac{\partial \theta}{\partial \xi}$ and $\frac{\partial \phi}{\partial \xi}$

were evaluated to a first-order finite difference approximation using the present value of $\xi$ (unknown) and the previous value of $\xi-\Delta \xi$ (known), with the unknown present value moved to the left hand side of Eq. (28).

The numerical results are affected by the number of mesh points in both directions. To obtain accurate results, a mesh sensitivity study was performed. After some trials, in the $\eta$-direction 190 mesh points were chosen whereas in the $\xi$-direction, 41 mesh points were used. The tolerance for convergence was $10^{-6}$. Increasing the mesh points to a larger value led to identical results.

The two systems of partial differential Eqs. (6)-(8) and (16)-(18) have similar form. Thus, they were solved using the procedure described above. The complete solution for the entire mixed convection regime was constructed from the two separate solutions of these two sets of equations.

\section{4}

\section{Results and discussion}

Numerical results for $\theta^{\prime}(\xi, 0)$ were obtained for $n$ ranging from 0.5 to 2 . In order to assess the accuracy of the numerical results, we compare our results for Newtonian fluid $(n=1)$ with those of Aldoss et al. [8] in Table 1. The agreement between the two is within $0.01 \%$ difference. Therefore, the present results are highly accurate. The velocity and temperature profiles are displayed in Figs. 2-7 for a range of values of $\lambda$ and $\xi_{f}$. The momentum and thermal boundary layer thicknesses increase as $\xi_{f}$ increases. The slip velocity at the porous surface $f^{\prime}(\xi, 0)$ decreases as the viscosity index $n$ increases. As the temperature exponent $\lambda$ increases, the momentum and thermal boundary layer thicknesses decrease. The surface temperature gradient and hence the heat transfer rate increases as $\xi_{f}$ increases. The slip velocity at the wall increases as $\xi_{f}$ increases.

Table 1. Values of $-\theta^{\prime}\left(\xi_{f}, 0\right)$ at selected values of $\xi_{f}$ for different $\lambda$ values

\begin{tabular}{lllll}
\multicolumn{5}{c}{$n=1.0$} \\
\hline$\xi_{f}=\mathrm{Ra}_{x}^{n} / \mathrm{Pe}_{x}^{(2 n+1) / 2}$ & $-\theta^{\prime}\left(\xi_{f}, 0\right)$ & & & \\
\cline { 2 - 5 } & $\lambda=0$ & $\lambda=0.5$ & $\lambda=1.0$ & $\lambda=2.0$ \\
\hline 0.001 & 0.565017 & 0.886622 & 1.128898 & 1.505421 \\
0.002 & 0.565018 & 0.886907 & 1.129397 & 1.506336 \\
0.01 & 0.565019 & 0.889173 & 1.133360 & 1.513569 \\
0.1 & 0.565020 & 0.913762 & 1.175208 & 1.587454 \\
0.2 & 0.566819 & 0.939423 & 1.216936 & 1.657948 \\
0.5 & 0.566841 & 1.007495 & 1.322737 & 1.828786 \\
1.0 & 0.571811 & 1.101340 & 1.461311 & 2.044616 \\
10 & 1.083938 & 1.838896 & 2.464401 & 3.549212 \\
100 & 4.020844 & 4.605979 & 5.403946 & 7.130854 \\
$\xi_{n}=\mathrm{Pe}_{x} / \mathrm{Ra}_{x}^{2 / 3}$ & $-\theta_{1}^{\prime}\left(\xi_{n}, 0\right)$ & & & \\
$0\left(\xi_{f}=\infty\right)$ & 0.428688 & 0.807698 & 1.091757 & 1.566769 \\
\hline
\end{tabular}

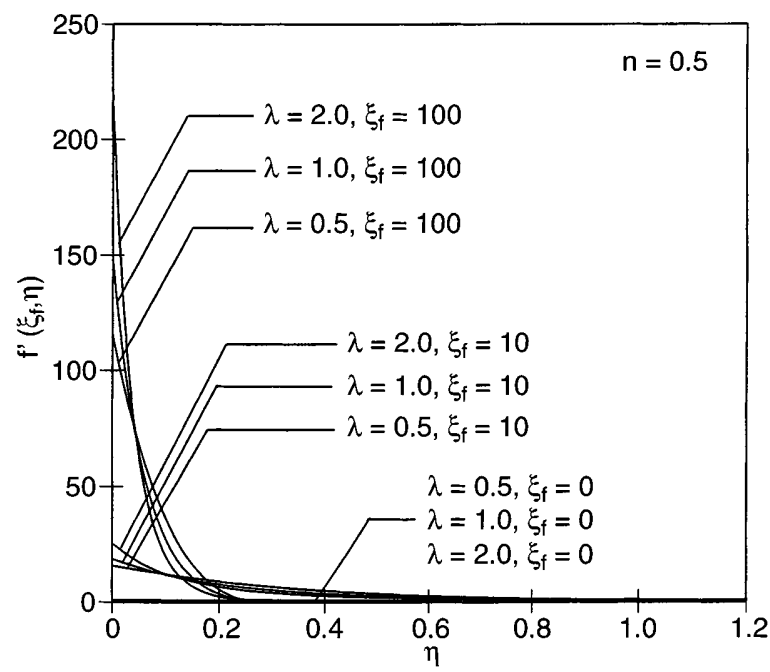

Fig. 2. Velocity distribution $(n=0.5)$

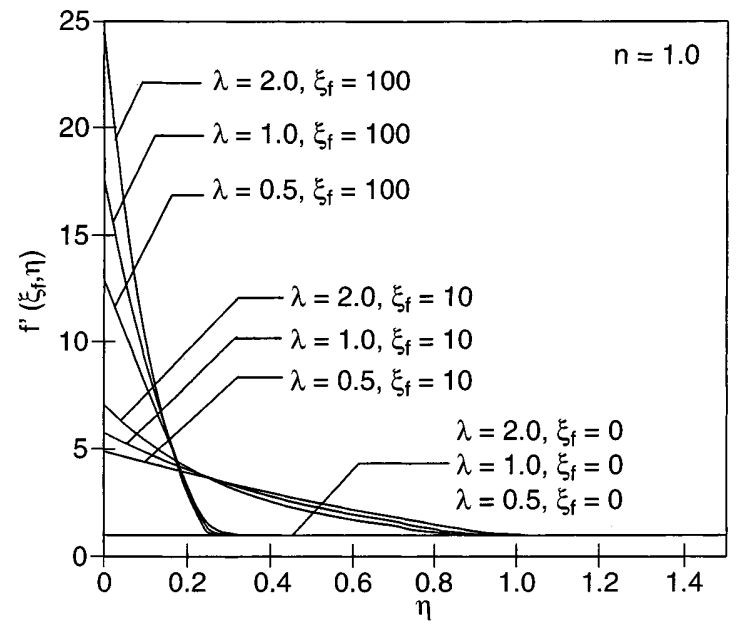

Fig. 3. Velocity distribution $(n=1.0)$ 


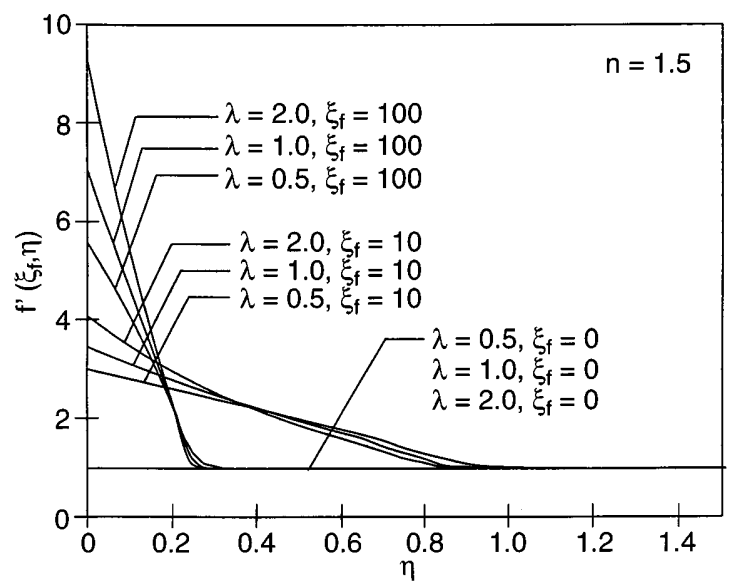

Fig. 4. Velocity distribution $(n=1.5)$

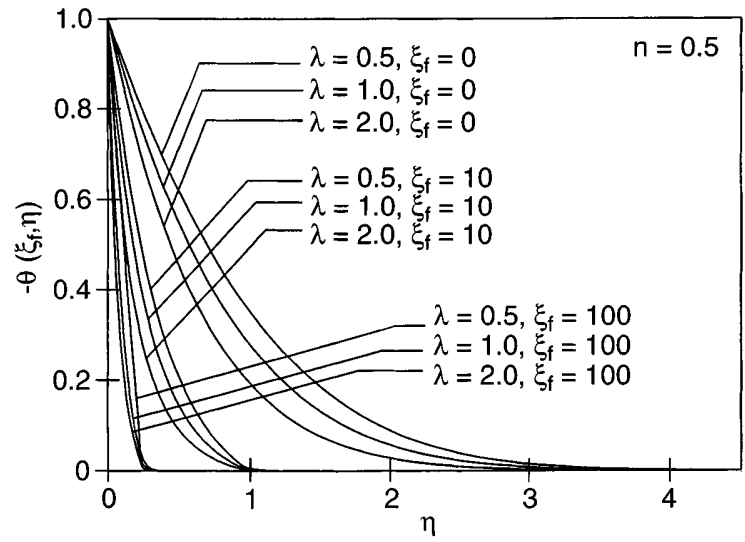

Fig. 5. Temperature distribution $(n=0.5)$

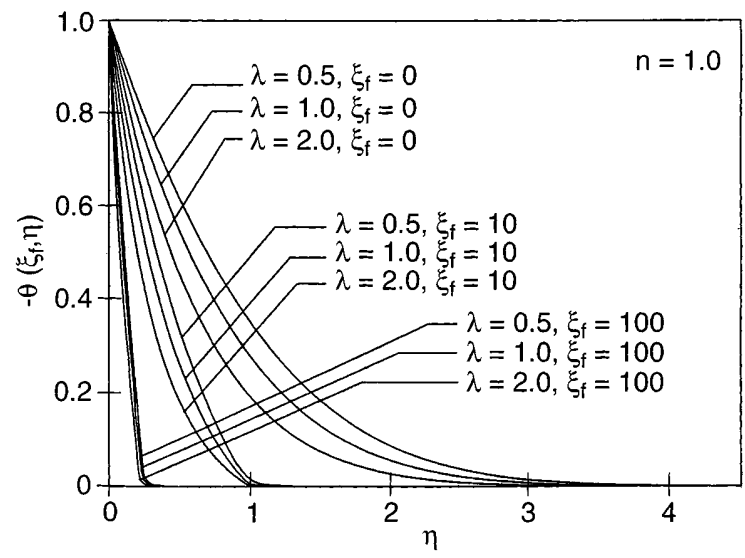

Fig. 6. Temperature distribution $(n=1.0)$

Figures 8-12 display the variation of Nusselt number with $\xi_{f}$ for $n$ ranging from $0.5-2.0$. The Nusselt numbers are normalized by the similarity solution results for each value of $n$. It is observed that the solutions for the forced convection dominated regime and the free convection dominated regime meet and match over the mixed convection regime. As $\lambda$ and $\xi_{f}$ increase, the Nusselt number increases for a given $n$. As $n$ increases, the Nusselt number

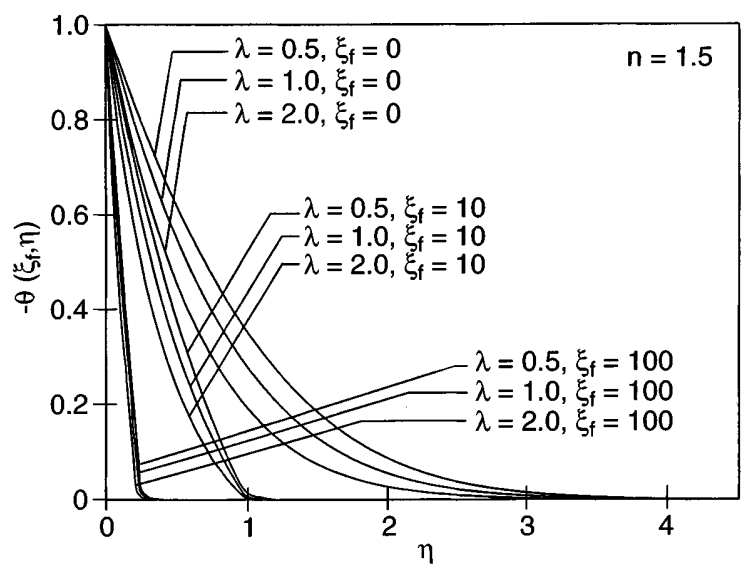

Fig. 7. Temperature distribution $(n=1.5)$

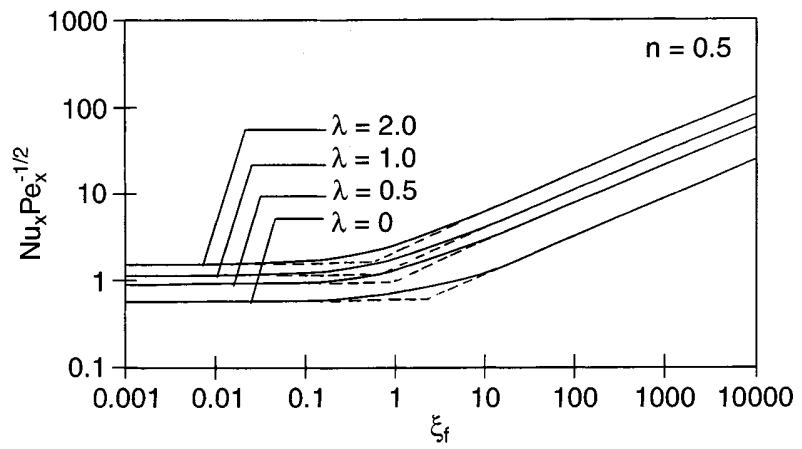

Fig. 8. Local Nusselt number variation $(n=0.5)$

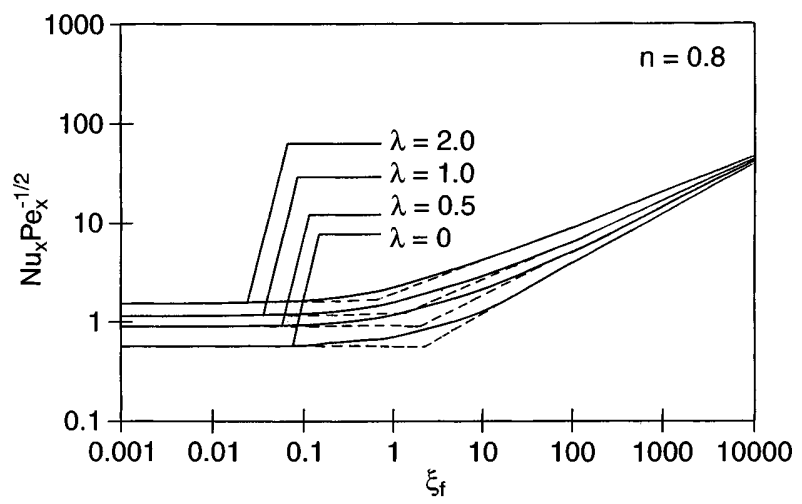

Fig. 9. Local Nusselt number variation $(n=0.8)$

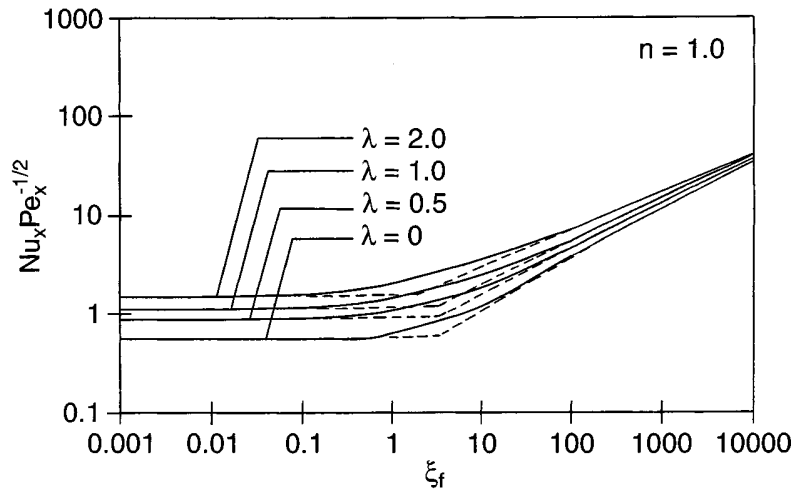

Fig. 10. Local Nusselt number variation $(n=1.0)$ 


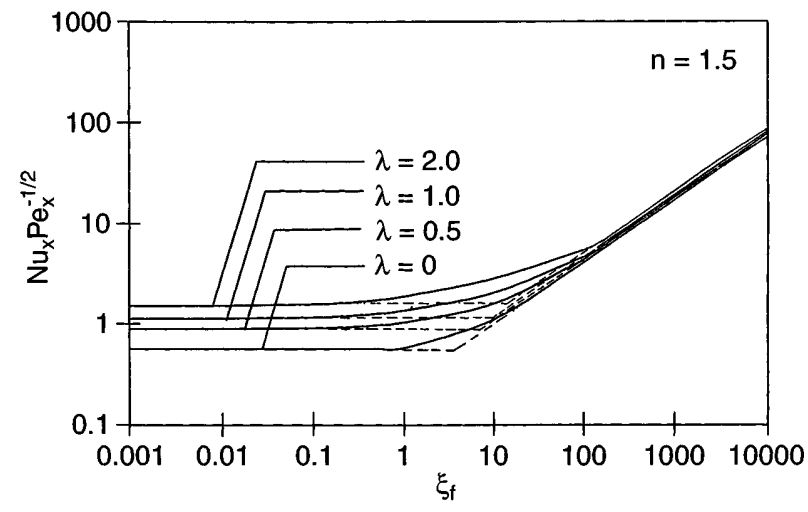

Fig. 11. Local Nusselt number variation $(n=1.5)$

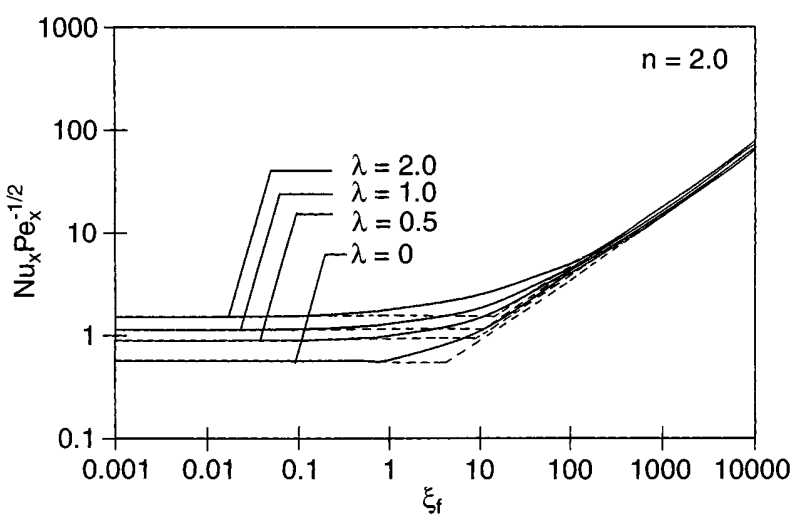

Fig. 12. Local Nusselt number variation $(n=2.0)$

Table 2. Domains of pure forced convection, mixed convection, and pure free convection

\begin{tabular}{llll}
\hline $\begin{array}{l}\text { Exponent } \\
n\end{array}$ & \multicolumn{2}{l}{ Range of $\xi_{f}=\mathrm{Ra}_{x}^{n} / \mathrm{Pe}_{x}^{(2 n+1) / 2}$} & values for: \\
\cline { 2 - 4 } & $\begin{array}{l}\text { Forced } \\
\text { convection }\end{array}$ & $\begin{array}{l}\text { Mixed } \\
\text { convection }\end{array}$ & $\begin{array}{l}\text { Free } \\
\text { convection }\end{array}$ \\
\hline 0.5 & $0-0.18$ & $0.18-11$ & $11-\infty$ \\
0.8 & $0-0.14$ & $0.14-14$ & $14-\infty$ \\
1.0 & $0-0.11$ & $0.11-17$ & $17-\infty$ \\
1.5 & $0-0.07$ & $0.07-24$ & $24-\infty$ \\
2.0 & $0-0.06$ & $0.06-27$ & $27-\infty$ \\
\hline
\end{tabular}

increases. The domain for pure forced convection, mixed convection and pure free convection may be established from the present results based upon 5\% difference in the Nusselt number from pure forced to pure free convection limit. These values are listed in Table 2.

\section{5}

\section{Concluding remarks}

In this paper, we have presented a boundary layer analysis for the mixed convection in non-Newtonian fluids along a non-isothermal horizontal plate embedded in fluid-saturated porous medium. The flow regime was divided into forced convection dominated and natural convection dominated regions. In the forced convection dominated region, $\xi_{f}=\frac{\mathrm{Ra}_{x}^{n}}{\mathrm{Pe}_{x}^{\frac{(2+1)}{2}}}$ characterizes the buoyancy effect on forced convection where as $\xi_{n}=\frac{\mathrm{Pe}}{\mathrm{Ra}_{x}^{2 / 3}}$ is a measure of the effect of forced flow on free convection. Numerical solutions using a finite difference scheme were obtained for the flow and temperature fields. The viscosity index, $n$ was varied from $0.5-2.0$.

\section{References}

1. Cheng, P.: Similarity solutions For mixed convection from horizontal impermeable surfaces in saturated porous media. International Journal of Heat and Mass Transfer 20 (1977) 893-898

2. Cheng, P.: Mixed convection about a horizontal cylinder and a sphere in a fluid-saturated porous medium. International Journal of Heat and mass Transfer. 25 (1982) 1245-1247

3. Nakayama, A.; Koyama, H.: A general similarity transformation for combined free and forced convection flows within a fluid-saturated porous medium. Journal of Heat Transfer, 109 (1987) 1041-1045

4. Cheng, P.; Minkowycz, W. J.: Free convection about a vertical flat plate embedded in a porous medium with application to heat transfer from a dike. Journal of Geophysical Research 82 (1977) 2040-2049

5. Gorla, R. S. R.; Zinolabedini, A.: Free convection from a vertical plate with nonuniform surface temperature and embedded in a porous medium. Transactions of ASME, Journal of Energy Resources Technology 109 (1987) 26-30

6. Gorla, R. S. R.; Tornabene, R.: Free convection from a vertical plate with nonuniform surface heat flux and embedded in a porous medium. Transport in Porous Media, 3 (1988) 95-106

7. Pop, I.; Gorla, R. S. R.: Horizontal boundary layer natural convection in a porous medium with a gas. Transport in Porous Media 6 (1991) 159-171

8. Aldoss, T. K.; Chen, T. S.; Armaly, B. F.: Nonsimilarity solutions for mixed convection from horizontal surfaces in a porous medium-variable wall temperature. International Journal of Heat and Mass Transfer, 36 (1993) 471-477

9. Chen, H. T.; Chen, C. K.: Free convection of non-newtonian fluids along a vertical plate embedded in a porous medium. Transactions of ASME. Journal of Heat Transfer 110 (1988) 257-260

10. Nakayama, A.; Koyama, H.: Buoyancy induced flow of nonnewtonian fluids over a non-isothermal body of arbitrary shape in a fluid-saturated porous medium. Applied Scientific Research 48 (1991) 55-70 